\title{
Tenascin Expression Patterns and Cells of Monocyte Lineage: Relationship in Human Gliomas
}

\author{
Andres Kulla, M.D., Aive Liigant, M.D., Andres Piirsoo, Ph.D., Gerd Rippin, Sci.D., \\ Toomas Asser, M.D., Ph.D. \\ Departments of Pathology (AK) and Neurology and Neurosurgery (AL, TA), Institute of General and \\ Molecular Pathology (AP), University of Tartu, Tartu, Estonia; Institute of Statistics, University of \\ Mainz, Mainz, Germany (GP)
}

Stromal extracellular matrix (ECM) components are thought to play an important role in regulating invasion of human gliomas. Macrophages and microglial cells may heavily influence the integrity of the extracellular compartment of gliomas, and the affected ECM may play a key role in regulating migratory activity of both tumor cells and macrophages/ microglia. The aim of this investigation was to study immunohistochemically the expression patterns of four ECM components: fibronectin, laminin, collagen IV, and tenascin (TN) in human gliomas, with special attention to TN. Our main goal was to study the possible correlation between $\mathrm{TN}$ expression and macrophagic/microglial infiltration in gliomas. Altogether, 90 gliomas were studied. Tumors included 46 glioblastomas, 19 anaplastic gliomas, 22 low grade gliomas, and 3 pilocytic astrocytomas. Vascular TN prevailed in perinecrotic areas of glioblastomas, whereas interstitial TN was more often expressed distant from necrosis and in the ECM of anaplastic and low grade gliomas. Double staining with CD68 and anti-TN antibodies showed that macrophagic/microglial density was significantly higher in TN-positive areas of most of the glioblastomas and anaplastic gliomas, whereas microglial percentage from total number of CD68-positive cells was in most of the cases significantly higher in TN-negative areas. In addition, we saw a morphologically spatial correlation between higher densities of macrophagic/microglial infiltration and TN expression in perinecrotic areas in glioblastomas. Attachment of macrophages to $\mathrm{TN}$-positive basement membrane zones of newly formed stromal blood vessels was evident. On the basis of our results, we conclude that TN may play a crucial role in

Copyright (C) 2000 by The United States and Canadian Academy of Pathology, Inc.

VOL. 13, NO. 1, P. 56, 2000 Printed in the U.S.A.

Date of acceptance: July 22, 1999.

Address reprint requests to: Andres Kulla M.D., Department of Pathology,

Maarjamõisa Hospital of University of Tartu, Puusepa 8, Tartu 51014,

Estonia; fax: 372-7-448-106. regulating trafficking of cells of monocyte lineage in human gliomas.

KEY WORDS: Extracellular matrix, Glioma, Immunohistochemistry, Macrophages, Microglia, Tenascin.

Mod Pathol 2000;13(1):56-67

Gliomas are the major group of human central nervous system tumors that affect almost all of the age groups of both sexes (1). Malignant transformation of gliomas is accompanied by extensive proliferation of stromal blood vessels. This phenomenon is considered to be one of the most reliable markers of the anaplastic change in tumors of glial origin (2) and is used as one of the morphologic landmarks in grading of these tumors by neuropathologists $(2,3)$. Malignant progression accompanied by vascular proliferation inevitably leads to the increase of the stromal components, including extracellular matrix (ECM) proteins in gliomas. In addition, changes in the protein composition of ECM take place, and these are believed to play an important role in induction and regulation of glioma cell migration (4-6). Several different ECM proteins (collagen IV [CIV], fibronectin [FN], laminin [LN], vitronectin, and tenascin $[\mathrm{TN}]$ ) have been proposed to participate in this process; LN and TN are the most permissive substrates for glioma cell migration in vitro $(4,5,7)$.

$\mathrm{TN}$ is an extracellular oligomeric glycoprotein complex that participates in the adhesion of cells to the ECM similarly to FN (8). TN is composed of several distinct domains containing epidermal growth factor-like repeats, FN-type repeats, a segment of great homology with FN $(9,10)$, and shares both adhesive and counteradhesive effects $(11,12)$. $\mathrm{TN}$ is expressed in the developing central nervous system during embryogenesis (13-15). It has been found in healing wounds (16) and in the ECM of tumors (17) and has been demonstrated in vitro to function variously as an attachment factor (18), an antiadhesive substrate (19), or a migration-promoting 
factor (20). Specific roles suggested for TN include interference with FN activity $(21,22)$ and immunomodulation (23).

Recent studies have shown that TN is predominantly deposited in the ECM of gliomas of higher malignancy grades (24) and is strongly associated with hyperplastic blood vessels, which suggests its role in the neovascularization of malignant gliomas (25). Some investigators have demonstrated that in proliferating glioma vessels that express TN, FN becomes downregulated (24). Zagzag et al. (26) found that TN mRNA is prevalently expressed by endothelial cells of proliferating blood vessels in human astrocytomas. Considering the possible important role of $\mathrm{TN}$ in stromagenesis of malignant types of gliomas, radioactively labeled antibodies against TN have been used in Phase I clinical trials to treat patients with glioblastomas (27). Despite the interesting data revealed by these studies, the biologic significance of TN in human tumors of glial origin remains enigmatic.

Macrophages are cells of monocyte lineage that are able to induce vascular proliferation in tumors (28). In 1932, Del Rio-Hortega (29) recognized the plasticity of microglial cells in the brain and proposed that these cells are capable of transforming into brain macrophages. Indeed, later investigations supported this idea and have shown that brain macrophages that appear in different pathologic processes, including astroglial tumors, are derived from microglia (30-32). Macrophages and microglial cells show similar immunophenotypic characteristics. Immunohistochemically, both cell types can be identified using commercially available antibodies, such as anti-Ricinus communis agglutinin, Ham-56, and CD68 (33), underlining at the same time their common histogenetic origin from the cells of blood monocyte lineage.

Tumor associated macrophages are proposed to be able to change the content of the ECM, thereby stimulating the migration of endothelial cells in malignant tumors (34). Recently, it has been demonstrated that regulation of astrocytic TN is mediated by synergistic action of transforming growth factor $\beta 1$ and basic fibroblast growth factor, secreted by macrophages in vitro as well as after injury in vivo (35). Gullberg and co-workers (36) showed morphologically the correlation of TN expression with macrophagic invasion in Duchenne muscular dystrophy and myositis.

On the basis of these previous studies, the aim of the current investigation was to determine the expression patterns of four ECM components-FN, $\mathrm{LN}, \mathrm{CIV}$, and $\mathrm{TN}$-in human gliomas. We were particularly interested in investigating the relationship between TN expression and macrophagic/microglial infiltration (factors that both are supposed to be involved in tumor stromagenesis) and also perivascular lymphocytic cuffing in human gliomas.

\section{MATERIALS AND METHODS}

\section{Tumor Specimens}

Ninety glioma specimens obtained between 1992 and 1995 (inclusive) at the Department of Neurology and Neurosurgery of the University of Tartu were investigated. Paraffin-embedded tumor samples were collected at the Department of Pathology of Maarjamõisa Hospital of University of Tartu. Tumors were classified and graded histologically by a neuropathologist (AK) according to the current World Health Organization criteria (3) and based on conventional hematoxylin and eosin staining of paraffin sections. This was complemented in problematic cases by immunohistochemistry (anti-glial fibrillary acidic protein [GFAP] monoclonal antibody).

The following groups of gliomas were evaluated: glioblastomas (GIV; total number, 46); anaplastic astrocytomas, anaplastic oligodendrogliomas, and mixed anaplastic oligoastrocytomas (GIII; total number, 19); astrocytomas, oligodendrogliomas, and mixed oligoastrocytomas (GII; total number, 22); and pilocytic astrocytomas (GI; total number, 3 ). There were 13 prevalent small cell-type tumors in the group of glioblastomas; 7 of them showed pseudopalisading of tumor cells around necrotic areas. Overall, 36 glioblastomas exhibited necrosis of different extent in the biopsy tissue. In 34 glioblastoma specimens, typical, partly glomeruloid vascular proliferation was established. All three pilocytic astrocytomas showed mild to moderate, partly glomeruloid vascular proliferation but rather monotonous pilocytic cellular morphology. Perivascular lymphocytic infiltration (cuffing) was evident in 28 of all of the gliomas studied.

\section{Immunohistochemical Staining}

Immunohistochemical staining for TN, FN, CIV, LN, macrophages, and GFAP was performed using the streptavidin biotin (StreptABComplex/HRP Duet; DAKO, Carpinteria, CA) staining method (37). Paraffin sections of formalin-fixed ( $10 \%$ neutral buffered formalin) tissue samples were deparaffinized and rehydrated. To unmask the TN, FN, collagen, and macrophage-microglial epitopes, microwave treatment of sections was used as described by Boon and Kok (38). LN antigen retrieval was performed by using proteinase $\mathrm{K}$ (DAKO) diluted at 1:50 in $0.05 \mathrm{~m}$ Tris- $\mathrm{HCl}, \mathrm{pH}$ 7.5. Tissue sections were incubated for 5 min with 3\% hydrogen peroxide in methanol to block the endogenous peroxidase activity. Incubation with normal goat 
serum diluted in Tris-buffered saline (TBS) (1:5) for $20 \mathrm{~min}$ to reduce background as a result of the hydrophobic interaction was followed by incubation with primary antibody for $60 \mathrm{~min}$ at room temperature. The following primary antibodies were used: monoclonal mouse antibody against purified human TN (DAKO), diluted at 1:50; monoclonal mouse antibody against purified pepsin fragments of human type IV collagen (DAKO), diluted at 1:100; monoclonal mouse antibody against áchain of human LN (DAKO), diluted at 1:40; polyclonal rabbit antibody against human FN (DAKO), diluted at 1:7500; antimacrophage antibody CD68, monoclonal mouse antibody against Gaucher cells (DAKO), diluted at 1:100; and monoclonal mouse antihuman GFAP (DAKO), diluted at 1:75. Replacement of the primary antibodies with nonimmune mouse serum served as the negative control. After the tissue sections were washed with $0.05 \mathrm{M}$ Tris/ $\mathrm{HCl}$, incubation with biotinylated goat antibody to mouse/rabbit immunoglobulins for $20 \mathrm{~min}$ was performed. The tissue sections then were incubated with StreptABComplex/HRP for $20 \mathrm{~min}$. After the sections were rinsed in TBS, the chromogen solution of 3,3'-diaminobenzidine was applied. Sections were counterstained with hematoxylin and mounted in Entellan (Merck, Darmstadt, Germany). Repeated stainings were performed in cases with doubtful or absent staining with anti-TN antibody, to confirm the negative results. For immunohistochemical double staining, sequential staining with the StreptABComplex/HRP method for both steps was used. Treatment with $2.0 \mathrm{M} \mathrm{HCl}(\mathrm{pH} \mathrm{2.0)}$ between the two steps was performed to remove all of the unconjugated immune complexes from the first step. The second step was completed by using 3-amino-9-ethylcarbazole (DAKO) as the chromogen, nuclei counterstained with hematoxylin and mounted in Glycergel (DAKO). The following monoclonal antibodies were used in doublestaining procedures: anti-TN (first step) + antiGFAP (second step); anti-TN (first step) + antimacrophage antibody CD68 (second step). Doublenegative controls were always used in doublestaining procedures: one with substitution of primary antibody by nonimmune serum in the first step and another one with substitution of primary antibody by nonimmune serum in the second step of the procedure.

\section{Evaluation of TN Expression and Lymphocytic Cuffing in Glioma Specimens}

The extent of TN positivity was evaluated semiquantitatively by two investigators separately as follows: -, no TN expression; +, less than $10 \%$ of stromal blood vessels or of intervascular and intercellular interstitium with $\mathrm{TN}$ positivity;,++ 10 to
$50 \%$ of stromal blood vessels or of intervascular and intercellular interstitium with TN positivity; +++ , more than $50 \%$ of stromal blood vessels or of intervascular and intercellular interstitium with TN positivity. Using this semiquantitative scaling, the investigators evaluated vascular and interstitial TN positivity separately in the perinecrotic zones (zone width not more than one microscopic medium power field, magnification $200 \times$, from necrosis) and in areas distant from necrosis (more than one microscopic medium power field, magnification $200 \times$, from necrotic edge) in the group of glioblastomas (GIV). Only blood vessels with definite TN positivity, either diffusely in the vessel wall or in the basement membrane zone, were assessed to be TN positive. TN staining outside the basement membrane zone was evaluated as intervascular or intercellular (interstitial).

In the same group of tumors, necrosis was evaluated to be present $(+)$ or absent $(-)$; amount of $\mathrm{TN}$-positive rests of blood vessels in necrotic areas was evaluated semiquantitatively as follows: - , absent; + , few; ++ , moderate; +++ , many. In addition, in all cases, perivascular lymphocytic cuffing was evaluated separately in TN-positive and TNnegative areas: -, absent; +, lymphocytic cuffing around one blood vessel found; ++ , lymphocytic cuffing around more than one blood vessels found.

The cellular type of glioblastomas was evaluated morphologically to be prevalently of mixed or of giant cell type or prevalently of small cell type.

\section{Evaluation of Macrophagic and Microglial Infiltration Density in Glioma Specimens}

According to the amount of areas available for analysis, CD68-positive cells were counted in 8 to 15 medium power fields (magnification $200 \times$ ) with a Zeiss Axioskop (Carl Zeiss, Jena, Germany) microscope in every biopsy specimen that was double stained with antibodies against TN and CD68. Image analysis system Zeiss Prolifex 2.0 (VIDAS 2.1) Demo-version: PLEX M1D.MCR (Carl Zeiss, Oberkochen, Germany), allowing separate manual marking of counted macrophages and microglial cells, was used for this purpose. As no known specific antibodies for differentiating between human macrophages and microglial cells exist, these cells were separately counted according to their morphology. Macrophages were identified as CD68positive, round or slightly oval-shaped cells with abundant cytoplasm, whereas microglial cells were recognized by their bi- or multipolar tender cytoplasmic morphology and characteristic small ovalshaped nuclei. The number of CD68-positive macrophages and microglia was evaluated separately in the TN-positive areas (areas with TN-positive blood vessels and/or diffuse intercellular/intervascular 
TN positivity) and in the areas with TN-negative blood vessels and TN-negative interstitium (TNnegative areas). Care was taken not to count the cells closer than one high power field to the necrotic areas. Using a measuring grid (Carl Zeiss) with $1-\mathrm{mm}$ scale bar, the counting area was measured to be $0.084 \mathrm{~mm}^{2}$ allowing the establishment of cell density per $0.1 \mathrm{~mm}^{2}$.

The average total number of CD68-positive macrophages and microglial cells per $0.1 \mathrm{~mm}^{2}$ (infiltration density of CD68-positive cells) and separately the average number of microglial cells per $0.1 \mathrm{~mm}^{2}$ (microglial density) were calculated for each specimen as an average of all fields counted.

\section{Statistical Analysis of the Data}

Statistical Analysis System (SAS Version 6.12; SAS Institute, Cary, NC) was used for statistical analysis of the data. Paired and unpaired $t$ tests were used to compare differences in cellular density between the different areas of the same cases and between the different areas of different cases. Significance of differences between more than two different groups of the same or of different type was calculated using the analysis of variance with a significance level of $5 \%$. Spearman's correlation analysis was performed to evaluate the correlation between different characteristics analyzed. The null hypothesis was that no correlation existed between characteristics, and this hypothesis would be rejected whenever $P>.05$.

\section{RESULTS}

\section{Immunostaining of ECM Proteins}

FN, LN, and CIV were constantly expressed in almost all of the gliomas of different malignancy grades. FN showed diffuse staining in vessel walls in GI, GII, and GIII gliomas as well as in proliferating blood vessels of glioblastomas. Although in some of the glioblastomas there were also some $\mathrm{FN}$ negative stromal vessels of that type, we did not analyze the correlation between FN negativity and TN positivity in these vessels. In a few glioblastomas, some perivascular fusiform mesenchymal cells and only occasional tumor cells contained FN in their cytoplasm. In general, there was no intervascular or intercellular FN staining in all of the gliomas studied. LN and CIV showed weak to strong and constant expression mainly in the basement membrane zones of almost all of the stromal blood vessels.

Data concerning the semiquantitative evaluation of TN expression in gliomas are given in Table 1 . TN immunoreactivity was detected at variable intensities in gliomas of different malignancy grades. It was often present in the stromal blood vessels of glioblastomas, where it was found as diffuse staining of vessel walls or sometimes as staining of the basement membrane zone. Only vessels with this specific TN staining patterns were considered to be TN positive. Many of the proliferating and glomeruloid stromal vessels showed TN expression of various intensity in glioblastomas (Fig. 1A). Many of these vessels were present in the proximate zone around the necrotic areas (Fig. 1B). According to statistical analysis, there were significantly more $(P=.0004) \mathrm{TN}$-positive vessels around the necrotic areas than in areas distant from the necrosis (Table 1). In 23 glioblastomas, homogenization of $\mathrm{TN}$ positive material (obviously derived from necrobiotic TN-positive blood vessels) was present at the edge of necrosis. In 30 glioblastomas, TN-positive rests of necrotic blood vessels were seen inside the necrotic area, and this correlated well $(r=.68, P=$ .0001) with the presence of TN homogenization at the edge of necrosis. Also, gliomas with lower malignancy grades showed the same kind but less extensive vascular TN expression (Table 1). In the group of malignant gliomas (GIII), 4 of 19 cases (21.1\%) had clear but not widespread vascular TN expression. In the group of gliomas with malignancy grade GII, 2 of 22 cases $(9.1 \%)$ showed vascular TN positivity. Two of three pilocytic astrocytomas (GI) had vascular TN expression. One of these also had proliferating blood vessels, which showed strong TN positivity in their walls; in another, there was weak TN expression in stromal vessels of nonproliferating configuration. Statistically strong correlation was found to be present between the malignancy grade $(\mathrm{G})$ and vascular TN expression in gliomas $(r=.56, P=.0001)$.

In addition to vascular $\mathrm{TN}$, a specific pattern of immunoreaction was detected between the blood vessels and between the tumor cells, where often intensive TN was found to surround tumor cells proper (Fig. 1C). Often, diffuse extensive intercellular/interstitial TN staining was found in the areas distant from the necrosis in glioblastomas (Fig. 1D). Statistically, there was significantly more $(P=$ .0001) interstitial TN in these areas not confined to the necrosis than in the areas close to the necrosis. Although not that extensive as in glioblastomas, diffuse interstitial TN staining was present in gliomas of lower grades of malignancy (Fig. 1E). In three glioblastomas and two anaplastic gliomas, cytoplasmic TN was seen inside the tumor cells (Fig. 1C). Of 19 malignant gliomas (GIII), 12 (63.2\%) had diffuse intervascular and intercellular TN expression. Fifteen of 22 gliomas with malignancy grade GII (68.2\%) had diffuse intervascular and intercellular TN expression. In general, this diffuse interstitial TN expression had only statistically weak correlation to the malignancy grade $(r=.28, P=$ 
TABLE 1. Number of CD68-Positive Cells per $0.1 \mathrm{~mm}^{2}$, Percentages of Microglial Cells from Total Number of CD68-Positive Cells, and TN Expression in 90 Gliomas Examined

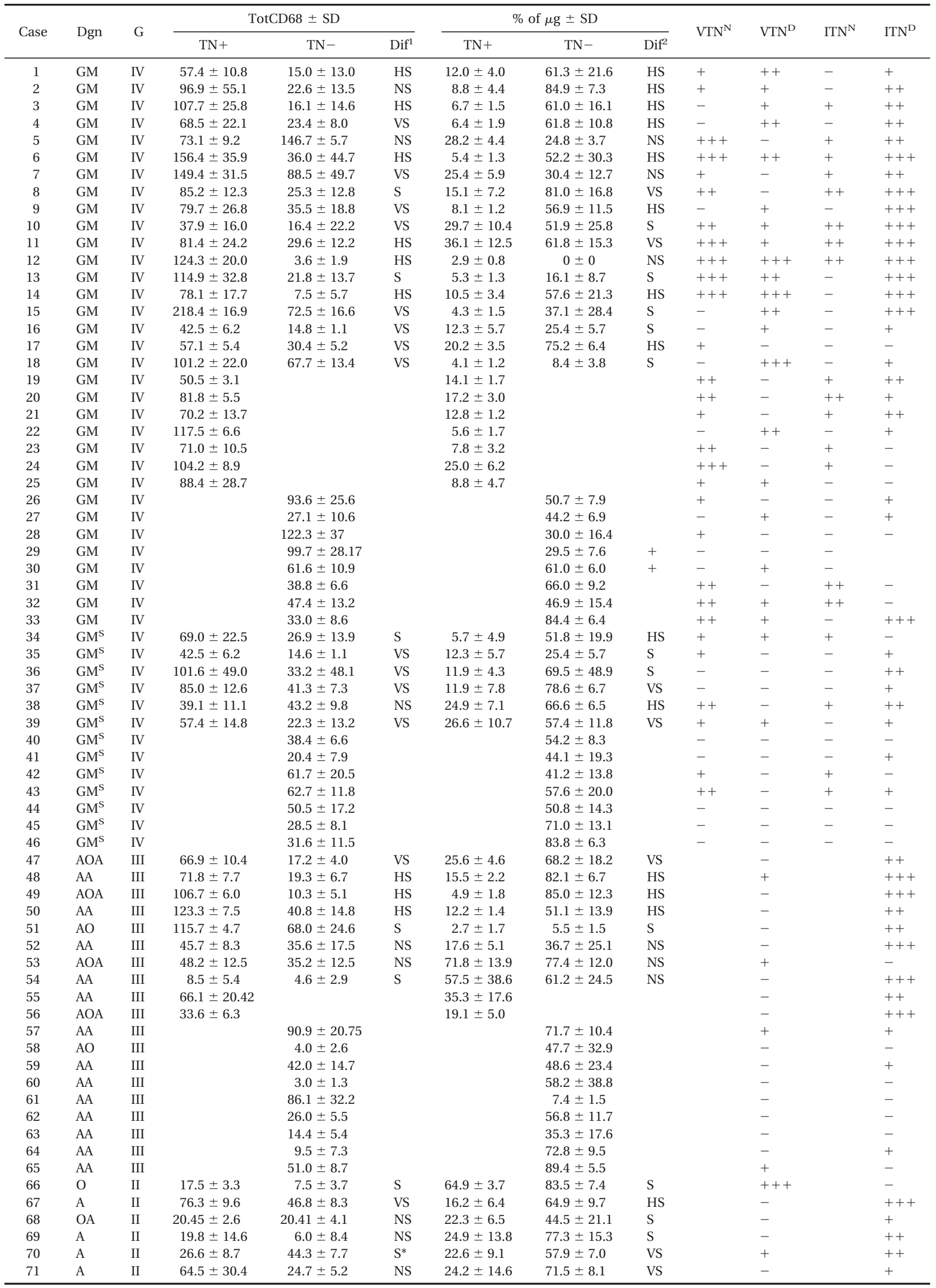


TABLE 1. (Cont.)

\begin{tabular}{|c|c|c|c|c|c|c|c|c|c|c|c|c|}
\hline \multirow{2}{*}{ Case } & \multirow{2}{*}{ Dgn } & \multirow{2}{*}{ G } & \multicolumn{3}{|c|}{ TotCD68 \pm SD } & \multicolumn{3}{|c|}{$\%$ of $\mu \mathrm{g} \pm \mathrm{SD}$} & \multirow{2}{*}{$\mathrm{VTN}^{\mathrm{N}}$} & \multirow{2}{*}{$\mathrm{VTN}^{\mathrm{D}}$} & \multirow{2}{*}{$\mathrm{ITN}^{\mathrm{N}}$} & \multirow{2}{*}{$\mathrm{ITN}^{\mathrm{D}}$} \\
\hline & & & $\mathrm{TN}+$ & $\mathrm{TN}-$ & $\operatorname{Dif}^{1}$ & $\mathrm{TN}+$ & $\mathrm{TN}-$ & $\mathrm{Dif}^{2}$ & & & & \\
\hline 72 & A & II & $107.9 \pm 12.5$ & $76.4 \pm 4.0$ & HS & $23.1 \pm 2.1$ & $71.0 \pm 4.2$ & HS & & - & & ++ \\
\hline 73 & A & II & $54.5 \pm 15.7$ & $55.6 \pm 5.9$ & NS & $21.0 \pm 2.9$ & $62.9 \pm 9.4$ & VS & & - & & ++ \\
\hline 74 & A & II & $50.1 \pm 4.7$ & $53.0 \pm 22.5$ & NS & $12.3 \pm 2.6$ & $12.7 \pm 5.1$ & NS & & - & & ++ \\
\hline 75 & A & II & $61.6 \pm 21.0$ & $34.0 \pm 15.0$ & NS & $23.4 \pm 5.3$ & $43.3 \pm 23.5$ & NS & & - & & +++ \\
\hline 76 & A & II & $40.4 \pm 5.6$ & & & $15.0 \pm 5.1$ & & & & - & & +++ \\
\hline 77 & A & II & $34.8 \pm 14.3$ & & & $39.8 \pm 28.8$ & & & & - & & ++ \\
\hline 78 & A & II & $57.1 \pm 10.0$ & & & $10.5 \pm 2.9$ & & & & - & & +++ \\
\hline 79 & A & II & & $5.2 \pm 2.1$ & & & $52.1 \pm 12.9$ & & & - & & + \\
\hline 80 & A & II & & $30.8 \pm 6.0$ & & & $84.4 \pm 9.7$ & & & + & & - \\
\hline 81 & $\mathrm{OA}$ & II & & $20.4 \pm 5.4$ & & & $42.1 \pm 22.1$ & & & - & & - \\
\hline 82 & $\mathrm{O}$ & II & & $42.3 \pm 12.5$ & & & $66.2 \pm 13.9$ & & & - & & + \\
\hline 83 & $\mathrm{OA}$ & II & & $20.4 \pm 5.4$ & & & $42.1 \pm 22.1$ & & & - & & - \\
\hline 84 & A & II & & $37.5 \pm 7.2$ & & & $77.3 \pm 7.7$ & & & - & & - \\
\hline 85 & A & II & & $9.0 \pm 6.7$ & & & $87.9 \pm 8.9$ & & & - & & + \\
\hline 86 & A & II & & $5.0 \pm 1.4$ & & & $93.1 \pm 11.1$ & & & - & & - \\
\hline 87 & A & II & & $26.8 \pm 7.4$ & & & $75.2 \pm 19.1$ & & & - & & - \\
\hline 88 & PA & I & $14.3 \pm 4.9$ & $18.7 \pm 3.9$ & $\mathrm{~S}^{*}$ & $15.3 \pm 7.5$ & $61.2 \pm 10.3$ & & & ++ & & - \\
\hline 89 & PA & I & & $6.7 \pm 3.0$ & & & $55.6 \pm 14.2$ & & & - & & - \\
\hline 90 & PA & I & & $16.8 \pm 15.1$ & & & $21.1 \pm 12.8$ & & & + & & - \\
\hline
\end{tabular}

Dgn, histopathologic diagnosis; GM, glioblastoma; $\mathrm{GM}^{\mathrm{S}}$, small cell glioblastoma; $\mathrm{AOA}$, anaplastic oligoastrocytoma; $\mathrm{AA}$, anaplastic astrocytoma; $\mathrm{AO}$, anaplastic oligodendroglioma; O, oligodendroglioma; A, astrocytoma; OA, oligoastrocytoma; PA, pilocytic astrocytoma; G, histologic malignancy grade; TotCD68, total number of CD68-positive cells per $0.1 \mathrm{~mm}^{2}$; \% of $\mu \mathrm{g}$, percentage of microglial cells from total number of CD68-positive cells per $0.1 \mathrm{~mm}^{2}$; $\mathrm{SD}$, standard deviation; $\mathrm{TN}+$, tenascin-positive areas; $\mathrm{TN}-$, tenascin-negative areas; Dif $^{1}$, difference between numbers of CD68-positive cells in TN-positive and TN-negative areas; Dif $^{2}$, difference between microglial percentages in TN-positive and TN-negative areas; HS, highly significant; NS, not significant; VS, very significant; S, significant; $\mathrm{S}^{*}$, inversely significant; VTN ${ }^{\mathrm{N}}$, vascular tenascin in perinecrotic areas; $\mathrm{VTN}^{\mathrm{D}}$, vascular tenascin in areas distant from necrosis and in GIII, GII, and GI gliomas; ITN ${ }^{\mathrm{N}}$, interstitial tenascin in perinecrotic areas; ITN ${ }^{\mathrm{D}}$, interstitial tenascin in areas distant from necrosis and in GIII, GII, and GI gliomas; -, no TN expression; +, less than 10\% of stromal blood vessels or of intravascular and intercellular interstitium with TN positivity;,++ 10 to $50 \%$ of stromal blood vessels or of intervascular and intercellular interstitium with TN positivity; +++ , more than $50 \%$ of stromal blood vessels or of intervascular and intercellular interstitium with TN positivity.

.0001). In addition to vascular and interstitial TN expression, there were areas with TN-negative blood vessels and interstitium in all malignancy grades of gliomas, including glioblastomas. TN was usually not found between the pseudopalisading tumor cells around the necrotic areas of glioblastomas. Moreover, in 13 of 46 glioblastomas (28.3\%), there was only weak or negative overall TN staining. Eight of these $13(61.5 \%)$ were morphologically of small cell type, often with tumor cell pseudopalisading around necrotic areas. In glioblastomas otherwise of more polymorphous cellular morphology but having areas consisting of small, primitive, tightly packed tumor cells, such small cell compartments did lack TN in their ECM. Often, the tumor cells of this type did not show any expression of GFAP. Statistically, there was strong negative correlation between this morphologic small cell type of glioblastomas and TN expression $(r=-.57, P=$ .001). In general, there was heterogenous TN expression throughout all of the malignancy grades as well as in individual cases. Twenty-nine biopsy specimens also contained peritumoral brain tissue. In $18(62 \%)$ of these cases, there was weak to moderate $\mathrm{TN}$ expression present in the nervous tissue surrounding the tumor (Fig. 1F). In four cases, that kind of TN staining was found in peritumoral brain tissue of gliomas of lower malignancy grade (GII).

\section{Cells of Monocyte Lineage (Macrophages and Microglia) and TN Expression}

Data concerning the medium values of the cell densities per $0.1 \mathrm{~mm}^{2}$ of CD68-positive macrophages and microglial cells in each separate case are given in Table 1. In all of the malignancy groups, only part of the tumors had both TNpositive and TN-negative areas available for counting CD68-positive cells. Of these, 15 (83.3\%) glioblastomas, 6 (75\%) anaplastic gliomas, and $3(30 \%)$ low grade (GII) gliomas had a significantly $(P<.05)$ higher number of CD68-positive cells per $0.1 \mathrm{~mm}^{2}$ in TN-positive areas than in TN-negative areas. Furthermore, 15 (83.3\%) glioblastomas, 5 (62.5\%) anaplastic gliomas, and 10 (80.0\%) low grade gliomas had a significantly higher percentage of microglial cells (from total number of CD68-positive cells) in TN-negative areas than in TN-positive areas.

The group of pilocytic astrocytomas (GI) contained only one case in which the CD68-positive cells could be counted in both TN-positive and TN-negative areas. In this pilocytic astrocytoma, the number of the CD68-positive cells per $0.1 \mathrm{~mm}^{2}$ was significantly $(P<.05)$ higher in TN-negative areas than in $\mathrm{TN}$-positive areas and the percentage of microglial cells from the total number of CD68positive cells was significantly higher in TNnegative areas compared with $\mathrm{TN}$-positive areas. 


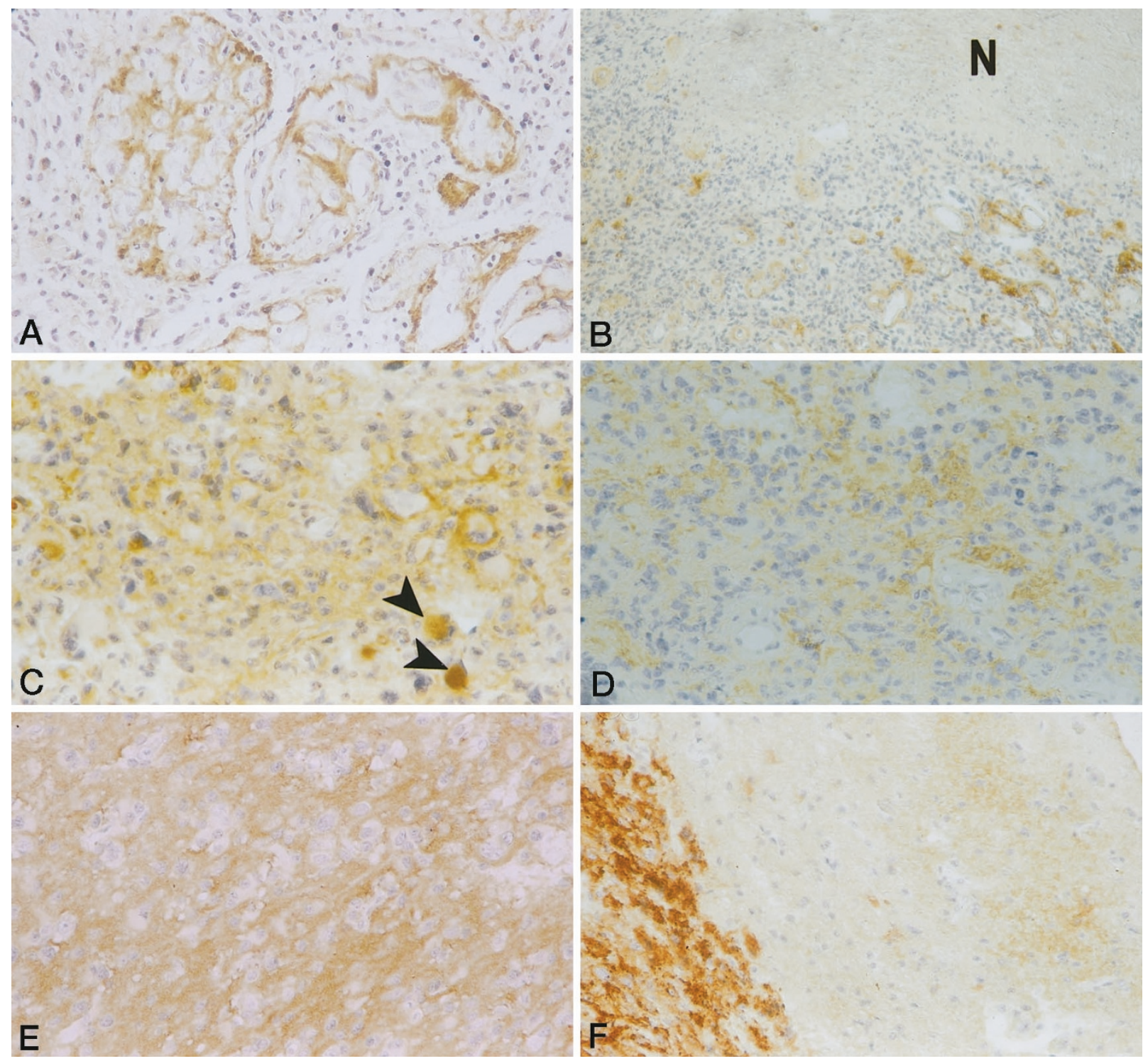

FIGURE 1. Tenascin (TN) immunoreactivity in gliomas (immunohistochemistry with anti-TN/3,3'-diaminobenzidine). A, glioblastoma. TN (light brown) is expressed in the walls and in the basement membrane zone of proliferating glomeruloid stromal blood vessels, $200 \times$. B, TN-positive stromal blood vessels surround an area of tumor necrosis $(\mathrm{N}), 200 \times$. C, TN is located around and between the tumor cells as well as in the cytoplasm of some of the tumor cells (arrowheads), 200×. D, diffuse intervascular and intercellular TN expression, 100 $\times$. E, intense diffuse TN expression in the extracellular matrix between the tumor cells in an oligoastrocytoma, $200 \times$. F, glioblastoma (brownish-red) infiltrating brain cortex. Diffuse TN expression (light brown) is present in the neuropil surrounding the tumor cells (double immunohistochemistry: anti-TN/3,3'diaminobenzidine + anti-glial fibrillary acidic protein/3-amino-9-ethylcarbazole), $200 \times$.

All cases, including those that did not have both TN-positive and TN-negative areas available for counting of CD68-positive cells (they had only TNpositive or only $\mathrm{TN}$ - negative areas available for cell counting), were used to analyze the density of CD68-positive cells in TN-positive and TN-negative areas in different malignancy groups in general.

Nonparametric analysis of variance showed that the medium values of the number of CD68-positive cells per $0.1 \mathrm{~mm}^{2}$ were significantly $(P=.0001)$ higher in TN-positive areas (Fig. 2A, B) compared with TN-negative areas in malignancy groups GIV, GIII, and GII, whereas there was no significant difference between these two characteristics in the GI group. The number of microglial cells per $0.1 \mathrm{~mm}^{2}$ as well as the percentage of microglial cells among the total number of CD68-positive cells was significantly higher in TN-negative areas compared with TN-positive areas in all malignancy groups (Fig. 2C). In general, the total number of CD68-positive cells per $0.1 \mathrm{~mm}^{2}$ (both in TN-positive and $\mathrm{TN}$ negative areas) tended to be higher in tumor groups with higher malignancy grade, whereas the number of microglial cells remained the same throughout all of the groups (Fig. 3). Density of CD68-positive cells and microglia in the group of glioblastomas of prevalently small cell morphology and with weak or absent TN expression was compared with the same characteristics of the rest of the glioblastomas and of the group of anaplastic (GIII) gliomas. The total 

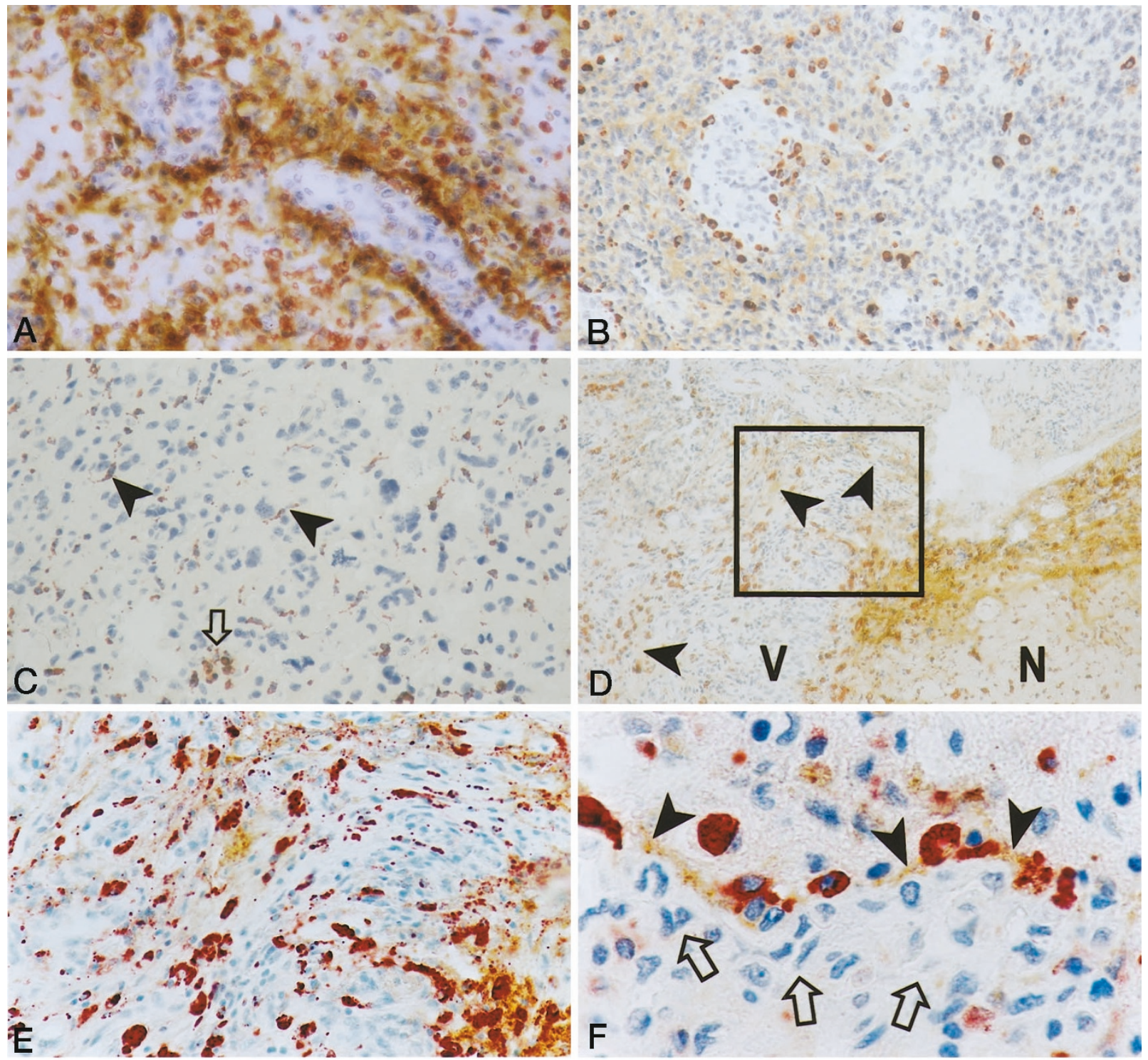

FIGURE 2. Tenascin (TN) (light brown) and CD68-positive macrophages/microglia (dark red) in gliomas (double immunohistochemistry: anti-TN/ 3,3'-diaminobenzidine + CD68/3-amino-9-ethylcarbazole). A, TN-positive area (vascular and interstitial TN expression) in a glioblastoma is heavily infiltrated by CD68-positive macrophages, 100×. B, a higher number of macrophages are present in the TN-positive area (left and middle) compared with TN-negative area (at right), where only occasional macrophages are present, 100×. C, TN-negative area in a glioblastoma. Most of the CD68positive cells are microglia (arrowheads). A small cluster of macrophages (empty arrow) are seen in the lower part of the figure, $200 \times$. D, glioblastoma. Streaming of macrophages via weak TN-positive extracellular matrix (arrowheads) toward necrosis (N). Edge of the necrotic area is showing homogenization of TN; a ribbon of newly forming stromal vessels (V) is seen at the edge of necrosis; no CD68-positive cells are present in this area, $100 \times$. E, higher magnification $(400 \times)$ from framed area from D. F, glioblastoma. Macrophages are adhering to TN-positive basement membrane zone (light brown) of newly forming stromal vessel tube (arrowheads), whereas in the opposite side, basement membrane zone is lacking TN (empty arrows) and there are no macrophages, $400 \times$.

number of CD68-positive cells in these small cell glioblastomas was significantly lower than in $\mathrm{TN}$ positive areas of the rest of the glioblastomas $(P=$ $.0001)$ and of the GIII gliomas $(P=.0001)$, whereas microglial density and percentage of microglial cells among the total number of CD68-positive cells were significantly higher in these small cell tumors than in TN-positive areas of the rest of GIV and of GIII gliomas.

Morphologically, a "streaming"-like pattern of CD68-positive macrophages via weakly TN-positive areas around the necrotic areas was seen in glioblastomas. In some cases, a TN-negative ribbon of primitive stromal vascular cells, obviously trying to form new vascular tubes, was seen at the edge of necrosis and was not infiltrated by macrophages. Macrophages where seen but in the areas where weak TN was present between these cells and where it fused into the homogenized TN-positive area at the necrotic edge. At this point, the necrotic areas contained a high number of macrophages (Fig. 2D, E). In a number of glioblastomas, adhesion 


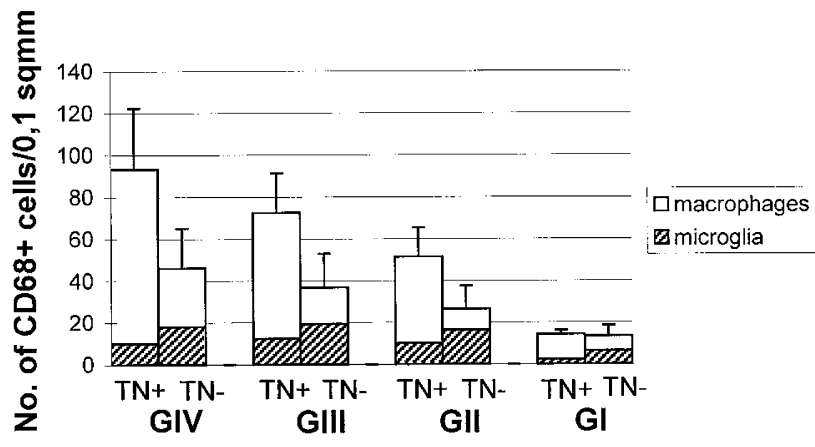

FIGURE 3. Density of CD68-positive cells in gliomas. Every bar represents total number of CD68-positive macrophages and microglial cells. Standard deviation is shown on the top of each bar. GI, GII, GIII, GIV, histologic malignancy grade.

of macrophages to the TN-positive basement membranes of newly forming stromal blood vessels was seen in glioblastomas (Fig. 2F).

\section{TN and Lymphocytic Cuffing}

In 28 cases of all of the gliomas studied, lymphocytic cuffing was found to be present. Fourteen of these 28 cases were glioblastomas, 6 were anaplastic gliomas (GIII), and 8 were low grade gliomas (GII). In 11 of these 28 cases, the lymphocytic cuffing was present in TN-positive areas; in 4 cases, it was present both in TN-positive and TN-negative areas. In the rest of 13 cases, the lymphocytic cuffing was seen in TNnegative areas of gliomas. Statistically, there was significantly $(P=.0042)$ more lymphocytic cuffing present in TN-negative areas than in TN-positive areas.

\section{DISCUSSION}

In the current study, we found that contrary to FN, $\mathrm{LN}$, and CIV, ECM protein TN showed heterogenous expression in human gliomas. TN expression patterns could be classified as vascular or interstitial types, and both were positively correlated to the malignancy grade of gliomas. These results are in accordance with data from previous studies (25). We found significantly more TN-positive blood vessels in close proximity to necrotic areas than in areas away from necrosis, and the reverse was true for interstitial TN expression. We also demonstrated that most of the glioblastomas that had only weak or no TN expression were morphologically of small cell type. In addition, using double-labeling immunohistochemistry, we found that higher densities of tumor infiltrating macrophagic/microglial cells correlated with stromal TN positivity in GIV, GIII, and GII gliomas, whereas the percentage of microglial cells was almost always higher in TN-negative areas of all malignancy groups. Some morphologic patterns seen in glioblastomas with macrophage streaming via $\mathrm{TN}$-positive areas around the necrosis and signs of adherence of mac- rophages to $\mathrm{TN}$-positive basement membranes of newly formed stromal blood vessels illustrate clearly the relationship between these two (extracellular and cellular) tumor components.

\section{TN Expression Patterns in Gliomas}

TN expression patterns described in our study were analogous to those described earlier $(24,25)$. We found less vascular TN to be present in GIII and GII groups of gliomas compared with a previous analysis (25). This can be due to differences in evaluating vascular TN expression. We did not consider blood vessels that were surrounded by TN to be TN positive if this protein was absent from the basement membrane zones or from the vessel walls. TN surrounding the blood vessels but lying outside the basement membrane zone proper was considered to be intervascular (interstitial). This may be the reason that most of the TN in GIII and GII gliomas in our series were evaluated as intercellular and intervascular. In addition, we found that many $\mathrm{TN}$-containing proliferating and newly formed blood vessels in glioblastomas were located in perinecrotic areas. In this region, tumor vasculature also has been shown to express vascular endothelial growth factor receptors (39). Vascular endothelial growth factor receptor itself is upregulated in these areas by hypoxia $(40,41)$. In our study, prevalence of TN-positive stromal vessels in close spatial relation to the necrosis suggests that vascular TN expression may as well be stimulated by hypoxia. This hypothesis may be supported by the widely known fact that TN is upregulated in vascular endothelial cells also during wound healing, and this always follows the necrosis of tissues by hypoxia (16). Contrary to vascular TN, in our study, interstitial TN expression seemed to be more extensive in areas away from necrosis in glioblastomas, and this type of TN was also present in peripheral areas of both malignant and benign gliomas. The peripheral areas of glioblastomas and especially of gliomas of lower grades of malignancy usually contained fewer blood vessels, and, therefore, it may be hypothesized that this type of extravascular TN could be synthesized by tumor cells rather than by endothelial cells. Zagzag and coworkers (26) showed that TN is mostly synthesized by endothelial cells in astrocytic tumors, and in only a few tumor cells, TN mRNA could be found using in situ hybridization. However, it can be argued that protein synthesis and secretion by the tumor cells have been completed and therefore the respective genes are no longer transcribed into mRNA. In some of the gliomas of our series, tumor cells contained TN in their cytoplasm. On that basis, it cannot be concluded whether the tumor cells produce or ingest $\mathrm{TN}$ from the surrounding ECM. In addition to interstitial $\mathrm{TN}$ expression by stromal 
ECM of gliomas, we found weak to moderate TN expression in peritumoral brain tissue in many cases, which contained the perifocal nervous tissue. In their confrontation experiments with threedimensional tumor spheroids against the spheroids from normal nervous tissue, Knott et al. (42) showed that glioma cells are capable of inducing the synthesis of ECM proteins in surrounding nonneoplastic brain tissue. It has been demonstrated that in tissue immediately surrounding the tumor and in infiltrated cortex, the neoformation of small blood vessels does not precede but follows the infiltration itself $(43,44)$. Therefore, considering the possible participation of $\mathrm{TN}$ in glioma cell migration, interstitial TN may seem to be engaged in this process first. This kind of promotion of tumor cell migration is hard to demonstrate in vivo. However, keeping in mind the dual counteradhesive and adhesive properties of TN (12), it cannot be excluded that this protein may act as well as a defense barrier against migrating tumor cells into the surrounding brain tissue. Thus, as a result of its dual nature, TN my act as a modulator of tumor cell migration.

It is interesting that many glioblastomas consisting mostly of small primitive tumor cells (so-called small cell glioblastomas) demonstrated only weak or even absent TN expression in their ECM. The same was seen in small cell areas of glioblastomas that otherwise did have polymorphous cell morphology. This type of glioblastoma or glioblastoma area often has high mitotic activity. It has been shown that the appearance of anaplasia in astrocytomas and the active cell proliferation in glioblastomas are sustained by the progressive increase of a cell population rich in mitoses, with isomorphous nuclei and negative for GFAP $(45,46)$. In our study, the lack of GFAP in primitive tumor cells was accompanied by the absence of TN expression in the ECM of such tumors or tumor areas. Castellani and co-workers (47) showed that distribution of TN in malignant gliomas is not correlated to cell proliferation. It is known that proliferation and migration are two reciprocal processes in malignant tumors (4). TN expression patterns seen by us may therefore suggest that TN does not participate in the malignant transformation of gliomas but may be active in regulating tumor cell migration in these tumors. That TN expression correlates with the malignancy grades in gliomas does not automatically mean that TN would participate in anaplastic transformation of these tumors.

Considering the heterogenous TN expression in human gliomas and its absence in several glioblastomas, histologic determination of its presence in biopsy tissues should be included as an integral part in the planning of future therapeutic strategies using radioactively labeled antibodies against TN to treat patients with malignant gliomas.

\section{TN and Cells of Monocyte Lineage}

It has previously been shown that high grade gliomas reveal a higher degree of infiltration by macrophages and microglial cells than low grade gliomas (48-50). In our study, the same correlation was found. In addition, we demonstrated that considerably higher numbers of macrophagic/microglial cells were present in TN-positive areas than in $\mathrm{TN}$-negative areas in gliomas, whereas the percentage of microglial cells was significantly higher in TN-negative areas. On the one hand, this finding indicates the possibility that some of the microglial cells present in the TN-negative areas may be turned into participants of the larger pool of activated macrophages present in the TN-positive areas, in the way proposed for microglial activation by earlier investigators $(29,30)$. On the other hand, the total number of the CD68-positive cells in TNpositive areas was unproportionately high compared with the differences in microglial densities between TN-positive and TN-negative areas. Two mechanisms to explain this can be postulated: either microglial cells proliferate before becoming activated to macrophages, or recruitment of blood monocytes to tissue macrophages takes place in gliomas. Defective or immature structure of walls of proliferating stromal blood vessels (which may be characterized by TN expression) may well contribute to that recruitment. It has been shown that blood monocytes must attach to endothelial cells to become activated tissue-type macrophages (51). Attachment of macrophages to TN-positive basement membrane zones of proliferating stromal blood vessels of glioblastomas observed by us suggests that TN may participate in this kind of activation of blood monocytes in gliomas.

Definite pattern of streaming of macrophages via perinecrotic weak TN-positive ECM toward necrosis demonstrated by us in a number of glioblastomas strongly supports that TN may be a permissive substrate for macrophagic/microglial migration in these tumors. Glioma cell migration supporting properties of TN have been demonstrated in cell culture studies (5). This is difficult to demonstrate in vivo (e.g., in biopsy material). In this regard, macrophages and microglial cells, being a much more dynamic cell population compared with tumor cells, seemed to be an informative cell population evidencing the possible role of $\mathrm{TN}$ as supporting mobile activity of cells in vivo.

\section{TN and Lymphocytic Cuffing}

In our study, we observed most of the perivascular lymphocytic cuffing to be present in $\mathrm{TN}$ negative areas of gliomas. Thus, lymphocytic cuffing negatively correlated with TN expression in these tumors. Brooks and co-workers (52) found 
perivascular lymphocytic cuffing in less malignant areas of glioblastomas and demonstrated longer survival for patients with malignant gliomas that had such perivascular lymphocytic cuffing in tumor tissue. Later studies, however, have not confirmed such a correlation (53). It is believed that immunologic mechanisms that may lead to eradication of a tumor are defective in malignant types of gliomas (54-57). This may be due in part to defective antigen presentation by the cells that are usually responsible for antigen presentation, (e.g., macrophages in gliomas). It may be questioned whether TN could repress this kind of immunologic activity of macrophages. TN shares immunomodulatory activities by attaching monocytes and inhibiting monocyte attachment to FN (23). Gundersen et al. (58) demonstrated that TN fragments derived from proteolytically degraded $\mathrm{TN}$ severely affect and block T-lymphocyte adhesion and activation in vitro. It could be an aim of further study to reveal whether the macrophages present in TN-positive areas of gliomas are sufficiently armed with essential receptors necessary for antigen presentation.

\section{CONCLUSIONS}

On the basis of the results of the present investigation, we conclude that vascular TN expression is spatially related to perinecrotic areas in glioblastomas, whereas interstitial TN is not; higher numbers of tumor-infiltrating macrophagic/microglial cells are present in $\mathrm{TN}$-positive areas in human gliomas; TN serves as a permissive substrate for macrophage migration and may have a certain role in modulating and possibly in promoting trafficking of cells of monocyte lineage in malignant human gliomas. TN expression should not be evaluated as a malignancy-associated phenomenon in tumors but rather as an integral part of reparative processes (although severely disturbed in tumors), in which it may route migratory activity of different cell types, including monocytes and possibly the tumor cells. From this aspect, our results support the concept of Nagy and co-workers (59) that tumors behave like wounds that do not heal.

Acknowledgments: This study was supported by Estonian Scientific Fund grant 2966 and partly by Deutscher Akademischer Austauschdienst. The authors thank Tiina Noorsalu, who carefully prepared a number of immunohistochemical double stainings, and Anu Kõiver, who contributed to this work. We also thank Dr. Jürgen Bohl (Department of Neuropathology, University of Mainz, Germany) for his kind help in establishing the pathomorphologic diagnoses in some of our glioma specimens. We are also grateful to Professor Hans H. Goebel (Depart- ment of Neuropathology, University of Mainz, Germany) and Professor Reinhardt Büttner (Institute of Pathology, University of Aachen, Germany) for carefully reading and commenting on the manuscript.

\section{REFERENCES}

1. Mahaley MS Jr, Mettlin C, Natarajan N, Laws ER Jr, Peace BB. National survey of patterns of care for brain tumour patients. J Neurosurg 1998;71:826-36.

2. Daumas-Duport C, Scheithauer B, O'Fallon I, Kelly P. Grading of astrocytomas: a simple and reproducible method. Cancer 1988;62:2152-65.

3. Kleihues P, Burger PC, Scheithauer BW. WHO international histological classification of tumours: histological typing of the tumours of the central nervous system. 2nd ed. Berlin: Springer-Verlag; 1993.

4. Koochekpour S, Merzak A, Pilkington GJ. Extracellular matrix proteins inhibit proliferation, upregulate migration and induce morphological changes in human glioma cell lines. Eur J Cancer 1995;31A:375-80.

5. Giese A, Loo MA, Rief MD, Tran N, Berens ME. Substrates for astrocytoma invasion. Neurosurgery 1995;37:294-302.

6. Paulus W, Huettner C, Tonn JC. Collagens, integrins and the mesenchymal drift in glioblastomas: a comparison of biopsy specimens, spheroids and early monolayer cultures. Int J Cancer 1994;58:841-6.

7. Giese A, Loo MA, Norman SA, Treasurywala S, Berens ME. Contrasting migratory response of astrocytoma cells to tenascin mediated by different integrins. J Cell Sci 1996;109: 2161-8.

8. Schenk S, Chiquet-Ehrismann R. Extracellular matrix proteins. Tenascins Meth Enzymol 1994;245:52-61.

9. Gulcher JR, Nies DE, Alexakos MJ, Ravikant NA, Sturgill ME, Marton LS, et al. Structure of the human hexabrachion (tenascin) gene. Proc Natl Acad Sci U S A 1991;88:9438-42.

10. Sriramarao P, Bourdon MA. A novel tenascin type III repeat is part of complex of tenascin alternative splices. Nucleic Acids Res 1993;21:163-8.

11. Friedlander DR, Hoffmann S, Edelmann GM. Functional mapping of cytotactin: proteolytic fragments active in cellsubstrate adhesion. J Cell Biol 1988;107:2329-40.

12. Prieto AL, Edelmann GM, Crossin KL. Characterisation of multiple adhesive and counteradhesive domains in the extracellular matrix protein cytotactin/tenascin. J Cell Biol 1992;119:663-78.

13. Riou JF, Shi DL, Chiquet M. Expression of tenascin in response to neural induction in amphibian embryos. Development 1988;104:511-24.

14. Bronner-Fraser M. Distribution and function of tenascin during cranial neural crest development in chick. J Neurosci Res 1988;21:135-47.

15. Mackie EJ, Tucker RP, Halfter W, Chiquet-Ehrismann R, Epperlein HH. The distribution of tenascin coincides with pathways of neural crest migration. Development 1988;102: 237-50.

16. Mackie EJ, Halfter W, Liverani D. Induction of tenascin in healing wounds. J Cell Biol 1988;107:2757-67.

17. Koukoulis GK, Gould VE, Bhattacharyya A, Gould JE, Howeedy AA, Virtanen I. Tenascin in normal, reactive, hyperplastic, and neoplastic tissues: biologic and pathologic implications. Hum Pathol 1991;22:636-43.

18. Bourdon MA, Ruoslahti E. Tenascin mediates cell attachment through an RGD-dependent receptor. J Cell Biol 1989; 108:1149-55.

19. Chiquet-Ehrismann R, Kalla P, Pearson CA, Beck K, Chiquet M. Tenascin interferes with fibronectin action. Cell 1988;53: 383-90. 
20. Chiquet-Ehrismann R, Mackie EJ, Pearson CA, Sakakura T. Tenascin: an extracellular matrix protein involved in tissue interaction during fetal development and oncogenesis. Cell 1986;47:131-9.

21. Lotz MM, Bursdal CA, Erickson HP, McClay DR. Cell adhesion to fibronectin and tenascin: quantitative measurements of initial binding and subsequent strengthening response. J Cell Biol 1989;109:1795-805.

22. Deryugina EI, Bourdon MA. Tenascin mediates human glioma cell migration and modulates cell migration on fibronectin. J Cell Sci 1996;109:643-52.

23. Ruegg CR, Chiquet-Ehrismann R, Alkay SS. Tenascin, an extracellular matrix protein, exerts immunomodulatory activities. Proc Natl Acad Sci U S A 1989;86:7437-41.

24. Higuchi M, Ohnishi T, Arita N, Hiraga S, Hayakawa T. Expression of tenascin in human gliomas: its relation to histological malignancy, tumor dedifferentiation and angiogenesis. Acta Neuropathol 1993;85:481-7.

25. Zagzag D, Friedlander DR, Miller DC, Dosik J, Cangiarella J, Kostinovsky M, et al. Tenascin expression in astrocytomas correlates for angiogenesis. Cancer Res 1995;55:907-14.

26. Zagzag D, Friedlander DR, Dosik J, Chikramane S, Chan W, Greco MA, et al. Tenascin-C expression by angiogenic vessels in human astrocytomas and by human brain endothelial cells in vitro. Cancer Res 1996;56:182-9.

27. Bigner DD, Brown MT, Friedman AH, Coleman RE, Akabani G, Friedman HS, et al. Iodine-131-labeled antitenascin monoclonal antibody 81C6 treatment of patients with recurrent malignant gliomas: phase I trial results. J Clin Oncol 1998;16:2202-12.

28. Polverini PJ, Leibovich SJ. Induction of neovascularisation in vivo and endothelial proliferation in vitro by tumour associated macrophages. Lab Invest 1984;51:635-42.

29. Del Rio-Hortega P. Microglia. In: Penfield W, ed. Cytology and cellular pathology of the nervous system. Vol 2. New York: Hoeber; 1932. pp. 481-534.

30. Streit WJ, Graeber MB, Kreutzberg W. Functional plasticity of microglia: a review. Glia 1988;1:301-7.

31. Streit WJ, Kreutzberg GW. Response of endogenous glial cells to motor neuron degeneration induced by toxic ricin. J Comp Neurol 1988;268:248-63.

32. Rossi ML, Hughes JT, Esiri MM, Coakham HB, Brownell DB. Immunohistochemical study of mononuclear cell infiltrates in malignant gliomas. Acta Neuropathol (Berlin) 1987;74: 269-77.

33. Hulette CM, Downey BT, Burger PC. Macrophage markers in diagnostic neuropathology. Am J Surg Pathol 1992;16:493-9.

34. Sunderkötter C, Steinbrink K, Goebler M, Bhardwaj R, Sorg C. Macrophages and angiogenesis. J Leukoc Biol 1994;55:410-22.

35. Smith GM, Hale H. Macrophage/microglia regulation of astrocytic tenascin: synergistic action of transforming growth factor-beta and basic fibroblast growth factor. J Neurosci 1997;17:9624-33.

36. Gullberg D, Velling T, Sjoberg G, Salmivirta K, Gaggero B, Tiger CF, et al. Tenascin expression correlates with macrophage invasion in Duchenne muscular dystrophy and myositis. Neuromuscul Disord 1997;7:39-54.

37. Shi ZR, Itzkowitz SH, Kim YS. A comparison of three immunoperoxidase techniques for antigen detection in colorectal carcinoma tissues. J Histochem Cytochem 1988;36:317-22.

38. Boon ME, Kok LP. Microwave cookbook of pathology: the art of microscopic visualisation. Leiden: Coulomb Press Leyden; 1987.

39. Chan AS, Leung SY, Wong MP, Yuen ST, Cheung N, Fan YW, et al. Expression of vascular endothelial growth factor and its receptors in the anaplastic progression of astrocytoma, oligodendroglioma and ependymoma. Am J Surg Pathol 1988; 22:816-26.

40. Plate KH, Breier G, Millaeur B, Ullrich A, Risau W. Upregulation of vascular endothelial growth factor and its cog- nate receptors in a rat glioma model of tumor angiogenesis. Cancer Res 1993;53:5822-7.

41. Stratmann R, Krieg M, Haas R, Plate K. Putative control of angiogenesis in hemangioblastomas by the von HippelLindau tumor suppressor gene. J Neuropathol Exp Neurol 1997;56:1242-52.

42. Knott JC, Mahesparan R, Garcia-Carbera I, Bolge Tysnes B, Edwardsen K, Ness GO, et al. Stimulation of extracellular matrix components in the normal brain by invading glioma cells. Int J Cancer 1998;75:864-72.

43. Levin V, Freeman M, Landahl H. The permeability characteristic of brain adjacent to intracerebral rat tumors. Arch Neurol 1975;32:785-91.

44. Schiffer D, Chiò A, Giordana MT, Mauro A, Migheli A, Vigliani MC. The vascular response to tumor infiltration in malignant gliomas: morphometric and reconstruction study. Acta Neuropathol (Berl) 1989;77:369-78.

45. Schiffer D, Giordana MT, Germano I, Mauro A. Anaplasia and heterogeneity of GFAP expression in gliomas. Tumori 1986;72:163-70.

46. Kunz J, Gottschalk J, Jänisch W, Schulz W. Zellproliferation und expression des säuren gliafaserproteins (GFAP) in hirntumoren. Acta Histochem 1986;80:53-61.

47. Castellani P, Sir A, Zardi L, Barbanera A, Dorcaratto A, Viale G. Distribution of tenascin in human malignant gliomas is not related to cell proliferation. J Neurol Neurosurg Psychiatry 1997;62:290-1.

48. Hitchcock ER, Morris CS. Mononuclear cell infiltration in central portions of human astrocytomas. J Neurosurg 1988; 68:432-7

49. Morris CS, Esiri MM. Immunocytochemical study of macrophages and microglial cells and extracellular matrix components in human CNS disease. 1. Gliomas. J Neurol Sci 1991; 101:57-85.

50. Roggendorf W, Strupp S, Paulus W. Distribution and characterisation of microglia/macrophages in human brain tumors. Acta Neuropathol (Berlin) 1996;92:288-93.

51. Judware R, Culp L. Extracellular matrix and matrix receptors: alteration during tumor progression. In: Bertino JR, ed. Encyclopedia of cancer. Vol 1. San Diego: Academic Press; 1997. pp. 660-79.

52. Brooks WH, Markesbery WR, Gupta GD, Roszman TL. Relationship of lymphocyte invasion and survival of brain tumor patients. Ann Neurol 1978;4:219-24.

53. Rossi ML, Jones NR, Candy E, Nicoll JA, Compton JS, Hughes $\mathrm{JT}$, et al. The mononuclear cell infiltrate compared with survival in high grade astrocytomas. Acta Neuropathol 1989; 78:189-93.

54. Gately CL, Muul LM, Greenwood MA, Papazoglou S, Dick SJ, Kornlith PL, et al. In vitro studies on the cell-mediated immune response to human brain tumors. J Immunol 1984; 133:3387-95.

55. Roszmann T, Elliott L, Brooks W. Modulation of T-cell function by gliomas. Immunol Today 1991;12:370-4.

56. Morioka T, Baba T, Black KL, Streit W. Inflammatory cell infiltrates vary in experimental primary and metastatic brain tumors. Neurosurgery 1992;30:891-6.

57. Morioka T, Baba T, Black KL, Streit WJ. Immunophenotypic analysis of infiltrating leukocytes and microglia in an experimental rat glioma. Acta Neuropathol 1992;83:590-7.

58. Gundersen D, Tran-Thang C, Sordat B, Mourali F, Ruegg C. Plasmin-induced proteolysis of tenascin C: modulation by $\mathrm{T}$ lymphocyte derived urokinase type plasminogen activator and effect on $\mathrm{T}$ lymphocyte adhesion, activation and cell clustering. J Immunol 1997;158:1051-60.

59. Nagy JA, Brown LF, Senger DR, Lanir N, Van de Water L, Dvorak HF. Pathogenesis of tumor stroma generation: a critical role of leaky blood vessels and fibrin deposition. Biochem Biophys Acta 1989;948:305-26. 